\title{
P13: Zum Zusammenhang zwischen kultureller Bildung und der Steigerung des Wohlbefindens bei Menschen mit Demenz
}

\author{
Franziska Fleischer · J. Hlawa \\ Nadine Fleischer-Schlechtiger $\cdot$ Matthias Ehrsam
}

Online publiziert: 23. Oktober 2013

(C) Springer-Verlag Wien 2013

Einleitung/Fragestellung: Pflegebedürftige Menschen mit einer Demenz benötigen Beschäftigungsangebote und Reize, um einer Isolation oder einer Deprivation entgegenzuwirken. Es bestehen verschiedene Möglichkeiten, bei Menschen mit Demenz durch Beschäftigungs- und Reizangebote, die Sinne anzuregen und eine drohende Isolation oder Deprivation zu verhindern oder zu mindern. Um den Bedürfnissen von Pflegebedürftigen zu entsprechen und somit das Wohlbefinden zu steigern, sind die Sinnesangebote gezielt, zum Teil zeitlich begrenzt und auf den Bewohner abgestimmt anzubieten. Das Hauptziel der vorliegenden Studie war die Steigerung des Wohlbefindens von Menschen mit Demenz durch medienpädagogische Projekte.

Zielstellung: Ziel der Bachelorarbeit war es, herauszufinden, ob ein Zusammenhang zwischen kultureller Bildung als Interventionsmaßnahme und der Steigerung des Wohlbefindens bei sieben Menschen mit einer diagnostizierten Demenz im Anfangsstadium besteht.

Methodik/Stichprobe: Die Untersuchung wurde in einer vollstationären Pflegeeinrichtung mit insgesamt 74 Bewohnern durchgeführt. Zur Ermittlung des Wohlbefindens der sieben Teilnehmer wurde eine in der Altenpflegeeinrichtung verwendete Wohlfühlskala eingesetzt, welche auf der
Grundlage des Well-being profile (Profil des Wohlbefindens) der Bradford Dementia Group erstellt wurde. Damit sollte anhand von 14 Indikatoren das Wohlbefinden, vor und nach den Workshops, mittels Prä- und Posttest durch teilnehmende Beobachtung eingeschätzt werden.

Ergebnisse: Insgesamt zeigte sich ein signifikanter Unterschied zwischen den eingeschätzten Prä- und Postwerten, woraus sich eine Steigerung des Wohlbefindens ableiten ließ. Da DCM und somit die Wohlfühlskala einen qualitativen Ansatz verfolgt und die Verhaltensdiversität und das Wohlbefinden im konkreten Setting des Workshops 1 und 2 abgebildet wurde, lassen die erhobenen Daten, Aussagen für die untersuchte Einrichtung zu. Trotz der guten Beobachtungssequenzen eigneten sich die Ergebnisse, aufgrund der Betrachtung von nur einer Einrichtung und sieben Menschen mit Demenz, nur eingeschränkt zur Generalisierung. Diskussion/Schlussfolgerungen: Die Durchführung der künstlerisch-kulturellen Veranstaltungen konnte die demenz-bedingten Symptome bei den Teilnehmern verringern. Daraus ließ sich ableiten, dass der Einsatz von Projekten kultureller Bildung, als Beschäftigung von Menschen mit Demenz im Anfangsstadium, eine Handlungsempfehlung für Einrichtungsleitungen darstellt.
F. Fleischer $\cdot$ J. Hlawa $\cdot$ M. Ehrsam

Fachbereich Soziale Arbeit. Medien. Kultur. Hochschule Merseburg, Merseburg, Deutschland

N. Fleischer-Schlechtiger

Volkssolidarität Leipziger Land/Muldental e. V., Leipzig,

Deutschland 